\title{
RADIO BURSTS WITH EXTRAGALACTIC SPECTRAL CHARACTERISTICS SHOW TERRESTRIAL ORIGINS
}

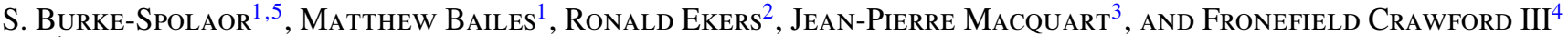 \\ ${ }^{1}$ Centre for Astrophysics and Supercomputing, Swinburne University of Technology, Mail H39, P.O. Box 218, Hawthorn VIC 3122, Australia; \\ sburke@astro.swin.edu.au \\ ${ }^{2}$ CSIRO Australia Telescope National Facility, P.O. Box 76, Epping NSW 1710, Australia \\ ${ }^{3}$ ICRAR/Curtin Institute of Radio Astronomy, GPO Box U1987, Perth WA 6845, Australia \\ ${ }^{4}$ Department of Physics and Astronomy, Franklin and Marshall College, Lancaster, PA 17604, USA \\ Received 2010 July 19; accepted 2010 October 29; published 2010 December 28
}

\begin{abstract}
Three years ago, the report of a solitary radio burst was thought to be the first discovery of a rare, impulsive event of unknown extragalactic origin. The extragalactic interpretation was based on the swept-frequency nature of the event, which followed the dispersive delay expected from an extragalactic pulse. We report here on the detection of 16 pulses, the bulk of which exhibit a frequency sweep with a shape and magnitude resembling the Lorimer Burst. These new events were detected in a sidelobe of the Parkes Telescope and are of clearly terrestrial origin, with properties unlike any known sources of terrestrial broadband radio emission. The new detections cast doubt on the extragalactic interpretation of the original burst, and call for further sophistication in radio-pulse survey techniques to identify the origin of the anomalous terrestrial signals and definitively distinguish future extragalactic pulse detections from local signals. The ambiguous origin of these seemingly dispersed, swept-frequency signals suggests that radio-pulse searches using multiple detectors will be the only experiments able to provide definitive information about the origin of new swept-frequency radio burst detections.
\end{abstract}

Key words: atmospheric effects - plasmas - pulsars: general - radio continuum: general

\section{INTRODUCTION}

Extragalactic radio phenomenon emitting transient radio bursts have been theorized to arise from a myriad of violent cosmic events, including coalescing systems of relativistic massive objects (Li \& Paczynski 1998; Hansen \& Lyutikov 2001), the evaporation of primordial black holes (Rees 1977), and supernova events (e.g., Colgate \& Noerdlinger 1971). The frequencydependent dispersive delay (at $\delta t \propto v^{-2}$ ) characteristic of a radio pulse that has propagated through the cold plasmas of the interstellar and intergalactic media encodes the source's distance and the line-of-sight free electron density, furthermore enabling extragalactic radio pulses to be used as robust cosmological probes of the ionized content of the intergalactic medium. However, such pulses have proven difficult to detect. There has thus far been a single claim for the detection of an extragalactic pulse: the discovery that has come to be called the "Lorimer Burst" (hereafter LB; Lorimer et al. 2007). The strongest evidence of its extragalactic origin was a large delay at $\delta t \propto v^{-2}$ (suggesting a dispersed signal, with a "dispersion measure," $\mathrm{DM}=375 \mathrm{pc} \mathrm{cm}^{-3}$, where the DM quantifies the integral electron density along the line of sight to the emitter), indicating a distance of well outside of the Galaxy when the electron content of our Galaxy is accounted for (Cordes \& Lazio 2002). It furthermore appeared to exhibit the frequency-dependent Komolgorov scattering that is expected from signals propagating in the interstellar medium. Based on its detection in three of the thirteen beams of the Parkes multibeam receiver at the expected relative levels for a point source, the burst appeared to be coming from the sky.

Since its discovery, some doubt has emerged over the extragalactic origin of the LB. Its extreme intensity $(\sim 100$ times the detection threshold) implies that searches of similar sensitivity should detect fainter events, if such bursts are isotropically

\footnotetext{
5 Also at CSIRO Australia Telescope National Facility, P.O. Box 76, Epping NSW 1710, Australia.
}

distributed throughout the universe and/or have an underlying intensity distribution typical of astrophysical phenomena. The failure of further searches to find similar events indicates that either this is not true, that the astrophysical process that caused the Lorimer Burst is extremely rare $\left(<2.8 \times 10^{-5} \mathrm{hr}^{-1} \mathrm{deg}^{-2}\right.$ for fluxes $S>300 \mathrm{mJy}$, based on the data from LB; Deneva et al. 2009; Keane et al. 2010; Burke-Spolaor \& Bailes 2010), or the burst's properties were misinterpreted.

We announce the detection of 16 pulses that exhibit a frequency-swept signal with similar characteristics to the Lorimer Burst. However, these new events are clearly of terrestrial origin with properties unlike any known sources of broadband radio emission. We detail the properties of the new detections and scrutinize them in comparison to the LB. We also discuss methods that can be used to distinguish an astrophysical or terrestrial origin for future detections of frequency-swept pulses.

\section{DATA SETS AND SEARCH}

Prompted by the discovery of the Lorimer Burst, we searched $1078 \mathrm{hr}$ of data for signals exhibiting a $\delta t \propto v^{-2}$ cold plasma dispersion delay. The data were archival pulsar surveys taken over the years 1998-2003 with the $20 \mathrm{~cm}$ multibeam receiver installed at Parkes Telescope. Four surveys were searched. For two (Edwards et al. 2001; Jacoby et al. 2009), we followed the search and inspection process described by Burke-Spolaor \& Bailes (2010), differing only in interference mitigation filters; we inspected a candidate if it either (1) was detected at a signal-to-noise ratio $(\mathrm{S} / \mathrm{N})$ of $>6$ in less than $9 / 13$ of the receivers, (2) had an $\mathrm{S} / \mathrm{N}$ of $\geqslant 20$, or (3) showed a DM of higher than $250 \mathrm{pc} \mathrm{cm}^{-3}$. As these were surveys of high Galactic latitude $\left(|b|>5^{\circ}\right)$, we searched DMs up to $600 \mathrm{pc} \mathrm{cm}^{-3}$. For the remaining two surveys (Crawford et al. 2006; Roberts et al. 2002, their observing parameters are detailed in their Section 2 and Table 1, respectively), we used methods described by (Crawford et al. 2007, 2009) applying no 


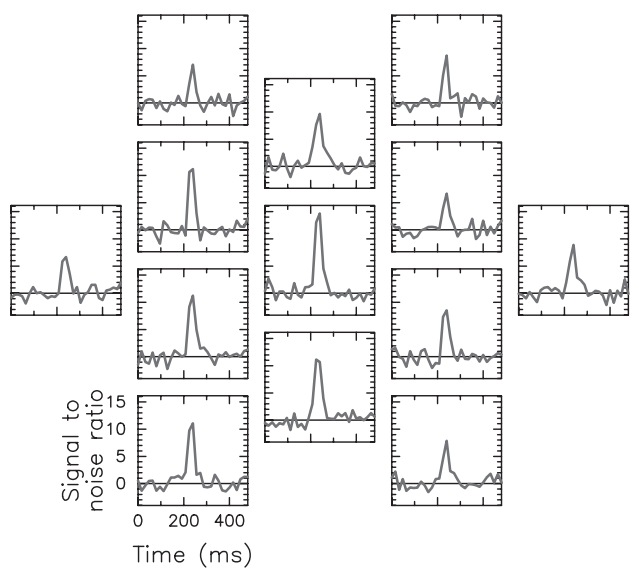

(a) Peryton 08 in 13 beams

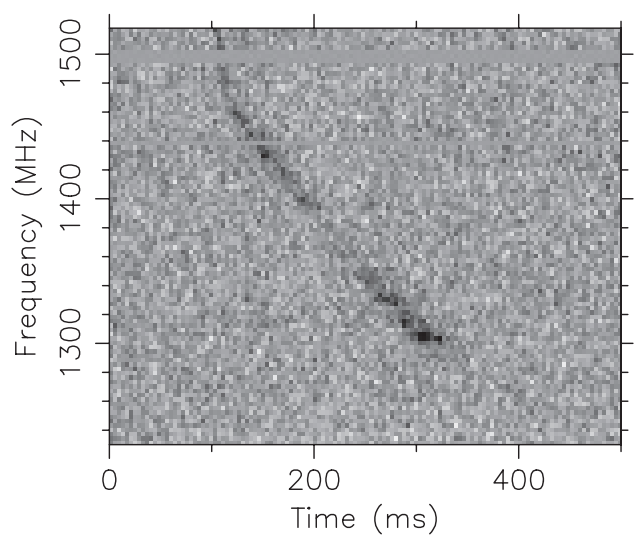

(c) Peryton 06

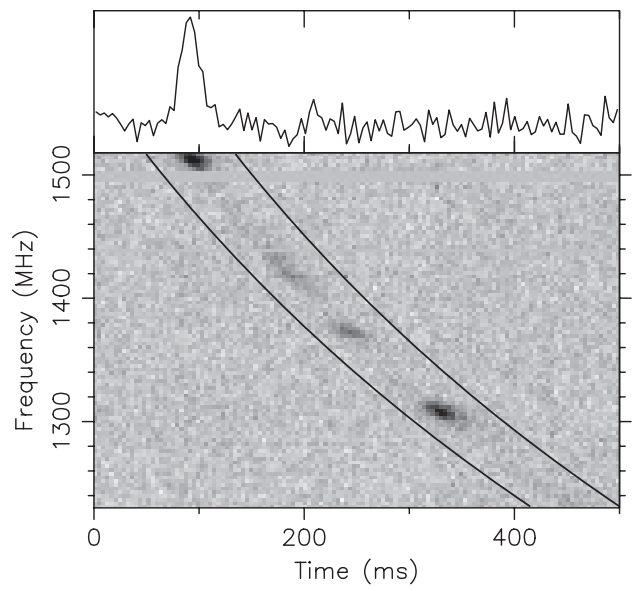

(b) Peryton 08

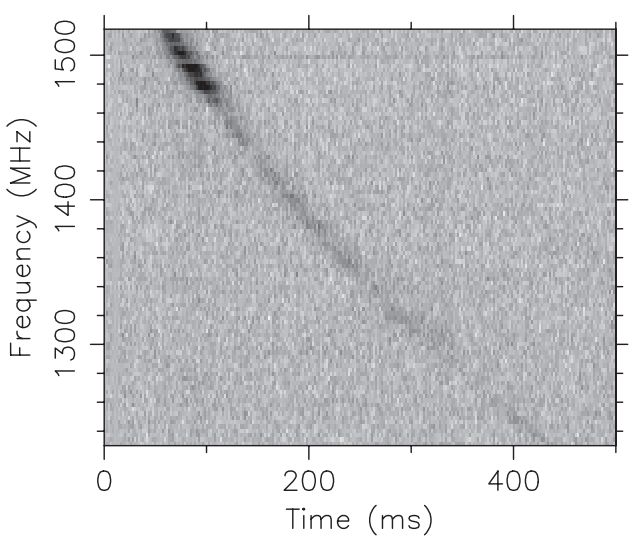

(d) Peryton 15

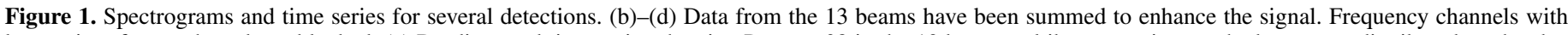

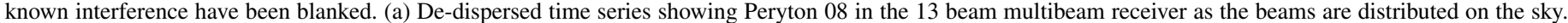

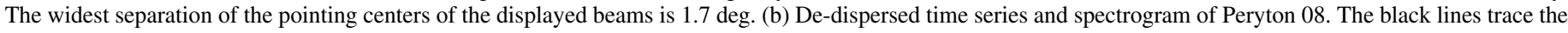
best-fit dispersive delay for this detection. (c) and (d) Spectrograms of Peryton 06 and 15, respectively.

interference excision based on multiple-beam detections. These were generally surveys of lower galactic latitude, therefore we searched DMs up to 1000 (Crawford et al. 2006), 2000 (for target AX J1826.11300 of the Roberts et al. survey), and $2500 \mathrm{pc} \mathrm{cm}^{-3}$ (for the other targets of Roberts et al.). Candidates from these surveys were inspected one beam at a time by eye to identify dispersed pulses.

\section{NEW DISCOVERIES AND THEIR PROPERTIES}

Our search revealed 16 pulses with two striking features that distinguish them from all others in the data: an apparent $\delta t \propto v^{-2}$ delay of a magnitude implying an extragalactic origin in the telescope's pointing direction, and a simultaneous occurrence in all 13 telescope receivers at relative intensities of less than a factor of four (Figure 1). When a dispersive delay is fit to each detection, the values cluster about a net band delay $\Delta t=360 \mathrm{~ms}$, indicating a close connection with the LB at $\Delta t=355 \mathrm{~ms}$ (Figure 2). The LB's reported sky position was below the horizon for several detections, therefore the pulses could not have come from the same extragalactic source. Below, we give evidence that the 16 signals have a terrestrial origin.

The 29' separation between each receiver's beam position and $>20 \mathrm{~dB}$ attenuation beyond $30^{\prime}$ from each beam center render it impossible for an on-axis, pointlike signal to appear in more than three beams at similar intensity (Staveley-Smith

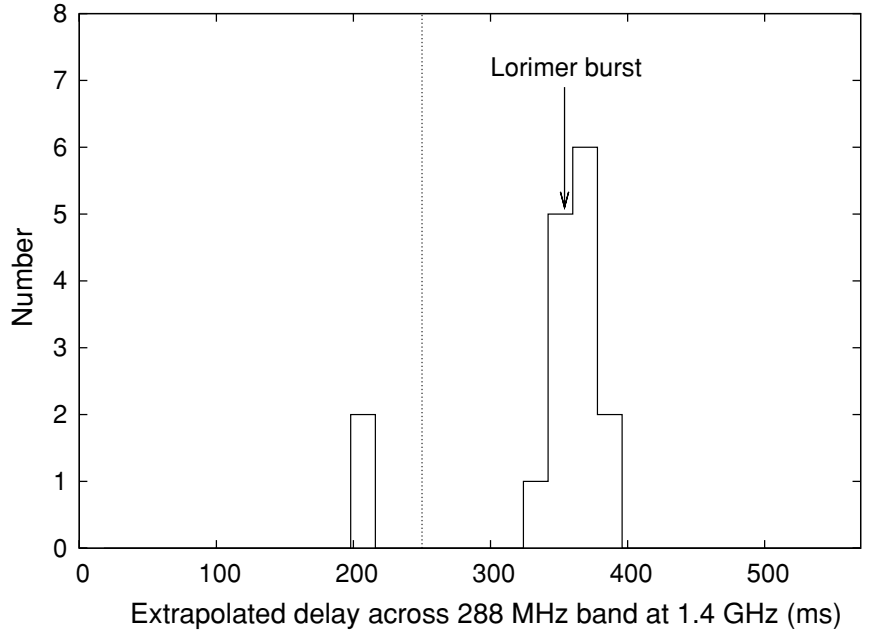

Figure 2. Distribution of fitted dispersive delays. The $x$-axis shows the total time to cross the observing band, calculated from each pulse as a best-fit dispersive sweep. An arrow indicates the delay of the Lorimer Burst. The dotted line indicates the delay below which we had uneven search criteria (see Section 2).

et al. 1996; Hunt \& Wright 1992). Out detections were therefore made through a sidelobe of the Parkes antenna, and based on the consistency of signals in the beams, in each case the emitter was positioned $\gg 5 \mathrm{deg}$ from the telescope's pointing 
Table 1

Detection Information and Measured Properties for Each Peryton and for the Lorimer et al. (2007) Burst

\begin{tabular}{lccccccc}
\hline \hline $\begin{array}{l}\text { Peryton } \\
\text { ID No. }\end{array}$ & $\begin{array}{c}\text { UT } \\
\text { (YY-MM-DD-h:m:s) }\end{array}$ & $\begin{array}{c}\theta_{z} \\
(\mathrm{deg})\end{array}$ & $\begin{array}{c}\theta_{a} \\
(\mathrm{deg})\end{array}$ & $\begin{array}{c}\Delta t \\
(\mathrm{~ms})\end{array}$ & $\begin{array}{c}\chi^{2} \\
(\mathrm{~ms})\end{array}$ & $\begin{array}{c}\mathbf{S}_{\mathrm{det}} \\
(\mathrm{mJy})\end{array}$ \\
\hline 01 & $98-06-23-02: 03: 44.91$ & 33.341 & 136.657 & 381.9 & 7.0 & 35.2 & 90 \\
02 & $98-06-23-02: 04: 06.75$ & 33.288 & 136.692 & 352.6 & 2.5 & 46.9 & 90 \\
03 & $98-06-23-02: 04: 28.84$ & 33.235 & 136.728 & 362.0 & 2.1 & 31.2 & 90 \\
04 & $98-06-23-02: 04: 36.84$ & 33.216 & 136.740 & 356.4 & 2.8 & 35.2 & 100 \\
05 & $98-06-23-02: 05: 17.77$ & 33.118 & 136.807 & 354.4 & 1.5 & 35.2 & 70 \\
06 & $98-06-23-02: 05: 39.50$ & 33.066 & 136.843 & 343.1 & 8.0 & 31.2 & 70 \\
07 & $98-06-23-02: 06: 01.81$ & 33.013 & 136.879 & 363.0 & 2.4 & 39.1 & 80 \\
$07 a$ & $98-06-23-02: 06: 24.13$ & 32.960 & 136.916 & 363.9 & $\ldots$ & 32.1 & 40 \\
08 & $98-06-23-02: 06: 31.89$ & 32.941 & 136.930 & 369.6 & 4.6 & 39.1 & 100 \\
09 & $98-06-23-02: 07: 27.70$ & 32.808 & 137.023 & 328.9 & $\ldots$ & 43.0 & 60 \\
10 & $98-06-23-02: 07: 49.78$ & 32.755 & 137.061 & 349.7 & 4.7 & 31.2 & 60 \\
11 & $98-06-23-02: 34: 53.63$ & 29.738 & 136.640 & 360.1 & 1.9 & 46.9 & 320 \\
12 & $98-06-25-05: 26: 49.13$ & 25.445 & 141.515 & 363.9 & 0.8 & 39.1 & 110 \\
13 & $02-03-01-01: 25: 38.88$ & 34.519 & 320.875 & 207.0 & 1.3 & 31.3 & 110 \\
14 & $02-06-30-02: 10: 29.38$ & 28.465 & 189.173 & 203.2 & 2.4 & 39.1 & 240 \\
15 & $03-07-02-00: 09: 23.96$ & 44.092 & 000.631 & 378.1 & 4.9 & 39.1 & 220 \\
\hline LB & $01-07-24-19: 50: 01.63$ & 42.419 & 183.315 & 354.5 & 1.6 & 15.6 & 30000 \\
\hline & & & & & & & \\
\hline
\end{tabular}

Notes. Columns: (1) chronological ID; Peryton 07a was discovered after summing the 13 beam data, therefore has a non-standard index. The Lorimer Burst is given for reference; (2) UT arrival time at 1516.5 MHz; (3, 4) telescope zenith and azimuth angles, respectively, at the time of detection; (5) extrapolated best-fit DM delay across the band; (6) reduced $\chi^{2}$ for a $\delta t \propto v^{2}$ fit to the burst, based on the event's time of arrival in $48 \mathrm{MHz}$ sub-bands (if $\mathrm{S} / \mathrm{N}_{\text {band }}>5$ ), timed against an analytic model of the event's de-dispersed profile at the best-fit DM. Events $07 \mathrm{a}$ and 09 had no sub-bands of $\mathrm{S} / \mathrm{N}_{\text {band }}>5$; (7) event width at half-maximum after de-dispersing at the best-fit quadratic delay; (8) detected single-beam peak flux, defined $S_{\mathrm{det}}=\mathrm{S} / \mathrm{N} \cdot T_{\text {sys }} /\left(G \sqrt{N_{\text {pol }} w B}\right)$, where $\mathrm{S} / \mathrm{N}$ is the detection's signal-to-noise ratio. The Perytons' intrinsic flux is much greater than reported here (see the text).

a This value would decrease without the presence of strong interference in the observation.

direction. Consequently: (1) we have a minimum-senisitivity field of view of $\sim 20000 \mathrm{deg}^{2}$, a detection rate of $2.3 \times$ $10^{-7} \mathrm{deg}^{-2} \mathrm{hr}^{-1}$ (0.1 day ${ }^{-1}$ with our observing system), and poor accuracy for emitter localization, (2) the pulses are subject to frequency-dependent dropouts and scattering from multi-path propagation-visible in Figures 1(b)-(d) and explored below, and (3) the source(s)' intrinsic flux density, had we pointed directly at it, is a factor of 2500-850000 greater than the detected value $\left(0.8 \mathrm{kJy}<S_{\text {intrinsic }}<272 \mathrm{kJy}\right.$ for the brightest detection, $0.1 \mathrm{kJy}<S_{\text {intrinsic }}<34 \mathrm{kJy}$ for the faintest; see Table 1$)$.

We conclude a terrestrial origin for these bursts based on their extreme brightness and two other features. First, some exhibit deviations from a model dispersive delay: e.g., the sharp kink at $1465 \mathrm{MHz}$ in detection 06 (Figure 1(c)), and subtler deviations in other detections (see the $\chi^{2}$ listing in Table 1). Despite a trend mimicking that expected from dispersion, such deviations decisively distinguish the pulses' frequency dependence from a delay induced by interstellar propagation. Hereafter, we distinguish these detections with the name "Perytons," representing the non-dispersive, highly swept, terrestrial signals exhibited by the pulses. ${ }^{6}$

Second, the temporal distributions of the signals strongly imply a terrestrial origin (Table 1). All detections occurred during daylight, primarily mid-morning. Eleven appeared in

\footnotetext{
6 The name is chosen from mythology to be unassociated with an exact physical phenomenon, due to the ambiguous origin of the detections; Perytons are winged elk that cast the shadow of a man.
}

one 4.4 minute observation followed by another $0.5 \mathrm{hr}$ later (we regard these as non-independent), while the remaining events occurred in isolation. Four of the five independent detections appeared in a three-week period in late June/early July spread across the years 1998-2003, coinciding with the peak of Australian mid-winter. Their time distribution appears to follow a non-random both annual and daily cycle; we tested the probability that $4 / 5$ events would occur in June/July given an underlying random distribution by running a Monte Carlo simulation based on the monthly hours observed. This test resulted in a confidence of $P=0.997231$ of a non-random annual distribution. A similar test based on the time of day distribution of observations and detections gave a probability of $P=0.999046$ that $4 / 5$ events would occur in the UT range $0-3$. These cycles are strongly suggestive of either a climate/ weather-related effect, or a man-made origin for the emission.

\section{DISCUSSION \\ 4.1. Signal Origins}

It is unprecedented for non-astrophysical emission to exhibit such drastic frequency-dependent delays in the $1 \mathrm{GHz}$ band. Given the daytime occurrence of the Perytons, we first explored the possibility of the signals as man-made. The continuous emission across the legally protected $1400-1427 \mathrm{MHz}$ band suggests that the signal is not intentionally transmitted; additionally, the lack of regular periodicity, broad pulse widths $(30-50 \mathrm{~ms})$, and broadband emission preclude a radar origin. Man-made emission that is unintentionally transmitted arises often from onsite electronic hardware failure. This does not appear to be the source of this emission, however, based primarily on the amplitude modulation seen in all the detections. These amplitudemodulated temporal or frequency structures show conformity across the spectrum in all 13 beams for each burst. Assuming the modulation is attributable to multi-path propagation effects (that these detections will necessarily show, as noted in Section 3 above), the incoming wavefront must not decorrelate over the physical size of the telescope's feed horns $(1 \mathrm{~m})$, to allow the similarity of the modulation structures in all 13 beams. The diffractive scale $\left(s_{0}\right)$, therefore, must likewise be $\geqslant 1 \mathrm{~m}$. Taking the characteristic bandwidth of the modulation structure in all pulses to be $\Delta f \sim 10 \mathrm{MHz}$ ), and based on the center frequency $f=1374 \mathrm{MHz}$, we place a lower limit on the distance to the object(s) and scatterer(s) of $2 \pi\left(s_{0} f\right) 2 / c \Delta f>4 \mathrm{~km}$. This suggests that we have detected emission from the horizon-well offsite from the telescope grounds - and provides the strongest argument against on-site hardware failure as the source. The quasiannual cycle and the spectral complexity with a 6 yr persistence of the signal also argue against local hardware failures as the emission's origin.

It is likewise possible that the Perytons were caused by a natural terrestrial source. We explored this possibility, considering processes that can produce non-dispersive, swept emission with sweep rates of $\sim 1 \mathrm{GHz} \mathrm{s}^{-1}$. The emission requires a process of finite-bandwidth $(\Delta f<25 \mathrm{MHz})$ signal to progressively change in its center frequency, for instance cyclotron emission in a time-varying magnetic field or the progressive incitement of plasma oscillations in regions of differing plasma density. The latter of these processes occurs in type III solar bursts (e.g., Loughhead et al. 1957), and it may be possible for such a process to occur in Earth's atmosphere with lightning or transient luminous event phenomena (e.g., Su et al. 2003; Sentman et al. 1995), which can reach the necessary ionization levels 
for Langmuir-wave electromagnetic emission to occur in our observing band.

\subsection{A Closer Look at the Lorimer Burst}

Whether the LB has the same origin as our detections remains inconclusive, despite the serious doubt these signals add to its extragalactic interpretation. Several qualitative differences between the LB and the Perytons warrant examination. The LB was clearly detected in only 3 of the 13 beams $(6,7$, and D). It is marginally detectable in other beams, most prominently in beam $\mathrm{C}$, and apparent in a stacked time series of the remaining beams. Based on observations of the inner sidelobe pattern of the multibeam obtained by L. Staveley-Smith (2010, private communication), we find a position of the LB (consistent with both the relative detected flux levels in beams $6,7, \mathrm{C}, \mathrm{D}$, and the non-detections in the other beams) at R.A. $19.44 \pm 0.08$, decl. $-75.17 \pm 0.08$. That is, the relative signal levels of the LB conform to those expected from a boresight signal, in agreement with the same conclusion of Lorimer et al. (2007). Therefore, if the LB is a Peryton, it appears to be the only detection for which the telescope was pointed directly at the emitter. Consequently, although the LB did not exhibit the same deep spectral signal modulation as the Perytons, these differences are well accounted for by the multi-path effects which we interpreted to arise from the horizon-based sidelobe detections of the Perytons (Section 4.1).

We note that because the beam and sidelobe shapes of the multibeam scale with frequency, the offset of the burst from the center of beam 6 will induce a spectral steepening of the source, causing the intrinsic spectrum to be flatter than that originally reported by Lorimer et al. (2007) by $\alpha_{\text {intrinsic }}=\alpha_{\text {observed }}-\alpha_{\text {induced }}$ (where $S \propto v^{\alpha}$ ). The Staveley-Smith measurements were made at two frequencies, allowing quantification of this effect. Within the error of our positional measurements, the induced index is $\alpha_{\text {induced }}=-1_{-2}^{+0.9}$. Therefore, at our estimate of position, the intrinsic spectral index is $\alpha_{\text {intrinsic }}=-2.5-0.6$, where we have measured the observed spectrum in beam 6 to follow $\alpha_{\text {obs }}=-2.6$.

If the LB was caused by a sky-based object (e.g., an aircraft or a natural, propagating swept-frequency phenomenon), we might expect to detect some movement of the LB across the field of the multibeam. We can limit the movement by noting that because the signal saturated beam 6 for the entirety of its sweep, it is clear that the emitter did not travel sufficiently far to cross a null in the sidelobes of beam 6 . Therefore, we limit any movement of the emitter to $<35$ arcmin, corresponding to a distance of $d=0.015 h / \sin (\theta)$, where $h$ is the emitter's altitude and $\theta$ is the angle between the telescope's line of sight and the LB's velocity vector. At $12 \mathrm{~km}$ (a typical aircraft/cloud height), this corresponds to a distance and velocity of $180 \mathrm{~m}$ and $500 \mathrm{~m} \mathrm{~s}^{-1}$, respectively, if the LB is moving perpendicularly to the line of sight. This does not put rigorous limits on aircraft movement, however, does place bounds on propagating atmospheric phenomena which could give rise to this emission. An analysis which places a more stringent limit on (or provides a measurement of) movement of the LB would be possible with a more sophisticated electromagnetic beam model.

One major point of discrepancy remains between the LB and the Perytons: that their widths disagree by a factor of $\sim 2$ (which cannot be accounted for by multi-path scatter broadening), and we do not observe a frequency-dependent pulse width evolution in the Perytons. However, we are hindered in measuring frequency-dependent evolution in the Perytons because of their modulated signal. While the difference in pulse width lends weak remaining support for a divide between the LB and the Perytons, with our current measurements it cannot be ruled out that there may be an underlying pulse width distribution, and/or a dependence on width with an event's intrinsic flux; likewise we cannot state whether frequency dependence of pulse width is intrinsic to the phenomenon causing all the events.

\section{CONCLUSIONS: IMPLICATIONS FOR CURRENT AND FUTURE TRANSIENT EXPERIMENTS}

Regardless of the physical origin of these pulses or the LB, the results of this study illustrate the limitations of singledish radio burst detection experiments to provide conclusive evidence for the origins of one-off bursts; dispersive delays can provide the only evidence for an astrophysical nature on single-detector detections, while multi-detector (i.e., array) experiments can provide wavefront measurement and localization for localized pulses, or can assure non-correlation of local signals between widely spaced array elements. Array experiments are necessary to provide a conclusive origin for further detections of the class of pulses presented here, as well as for any future experiments that aim to detect and use extragalactic pulses in scientific studies. Two such experiments are currently underway at the Giant Metre Wave and Very Long Baseline Array telescopes, and will be possible with the Square Kilometre Array and its pathfinder experiments. Single-dish measurements can improve our understanding of these events by providing polarization measurements, and further detections for world, temporal, and delay-distribution statistics.

If our detections do originate from a natural terrestrial process with intrinsic flux $\gg 100 \mathrm{Jy}$, detections are expected at other observatories with capabilities similar to Parkes and ongoing pulsar and transient observations (e.g., Arecibo Observatory, Green Bank, and Effelsberg Telescopes). It is an undeniable curiosity that no Perytons have yet been identified at radio observatories where such searches have been performed (e.g., Amy et al. 1989; Nice 1999; Deneva et al. 2009). However, it is possible that they have not occurred during observations (one should occur per $\sim 215 \mathrm{hr}$ of data on a telescope with similar system temperature and sidelobe suppression levels to Parkes, if the event rate is the same at other sites), that interference rejection algorithms based on the appearance of signals in multiple beams have removed the signals, that the searches were at frequencies where Perytons do not emit, and/or that the experiments were not sensitive to pulses of 30-50 ms width. As multi-detector coincidence filters will strongly preclude the detection of Perytons, the strength of such rejection filters to limit local interference of any form is clear; however, it is likewise clear that the filters would need to be relaxed to maximize an observatory's capability to detect and identify the origin of these remarkable signals. Additionally, we believe that it was the human inspection of the spectrogram data and multibeam time series for each candidate (described in Section 2) that were the main contributors to the first recognition of the peculiar nature of the Perytons- the simultaneous occurrence of clear, seemingly dispersed emission and multiple-beam detection-and that encouraged us to scrutinize these events more closely instead of disregarding them as spurious detections.

The authors gratefully acknowledge valuable discussions with M. Kesteven, C. Wilson, P. Burford, J. Reynolds, 
D. Melrose, P. Kalberla, and P. Edwards about various aspects of this research. Financial support was granted by the Research Corporation and the Mount Cuba Astronomical Foundation (F.C.), and the Australian Research Council (M.B.).

\section{REFERENCES}

Amy, S. W., Large, M. I., \& Vaughan, A. E. 1989, Proc. Astron. Soc. Australia, 8,172

Burke-Spolaor, S., \& Bailes, M. 2010, MNRAS, 402, 855

Colgate, S. A., \& Noerdlinger, P. D. 1971, ApJ, 165, 509

Cordes, J. M., \& Lazio, T. J. 2002, arXiv:astro-ph/0207156

Crawford, F., Hessels, J. W. T., \& Kaspi, V. M. 2007, ApJ, 662, 1183

Crawford, F., Lorimer, D. R., Devour, B. M., Takacs, B. P., \& Kondratiev, V. I. 2009, ApJ, 696, 574

Crawford, F., et al. 2006, ApJ, 652, 1499

Deneva, J. S., et al. 2009, ApJ, 703, 2259
Edwards, R. T., Bailes, M., van Straten, W., \& Britton, M. C. 2001, MNRAS, 326,358

Hansen, B. M. S., \& Lyutikov, M. 2001, MNRAS, 322, 695

Hunt, A., \& Wright, A. 1992, MNRAS, 258, 217

Jacoby, B. A., Bailes, M., Ord, S. M., Edwards, R. T., \& Kulkarni, S. R. 2009, ApJ, 699, 2009

Keane, E. F., et al. 2010, MNRAS, 401, 1057

Li, L. X., \& Paczynski, B. 1998, ApJ, 507, L59

Lorimer, D. R., Bailes, M., McLaughlin, M. A., Narkevic, D. J., \& Crawford, F. 2007, Science, 318, 777

Loughhead, R. E., Roberts, J. A., \& McCabe, M. K. 1957, Aust. J. Phys., 10, 483

Nice, D. J. 1999, ApJ, 513, 927

Rees, M. J. 1977, Nature, 266, 333

Roberts, M. S. E., et al. 2002, ApJ, 577, L19

Sentman, D. D., Wescott, E. M., Osborne, D. L., Hampton, D. L., \& Heavner, M. J. 1995, Geophys. Res. Lett., 22, 1205

Staveley-Smith, L., et al. 1996, PASA, 13, 243

$\mathrm{Su}, \mathrm{H}$. T., et al. 2003, Nature, 423, 974 